\title{
Hardware-in-the-loop Test Concept for an Energy-optimized Process Control
}

\author{
Tobias Kull' ${ }^{1}$ Bernd Zeilmann², Gerhard Fischerauer ${ }^{1}$ \\ ${ }^{1}$ Chair of Measurement and Control Systems and Center of Energy Technology (ZET), \\ University of Bayreuth, 95447 Bayreuth, Germany \\ ${ }^{2}$ Richter R\&W Steuerungstechnik GmbH, Körzendorf 52, 95491 Ahorntal, Germany \\ mrt@uni-bayreuth.de
}

\begin{abstract}
In this work, a hardware-in-the-loop test concept for the evaluation of energy-optimized process control is presented. Modern production facilities often contain distributed renewable energy systems and controllable loads. Coordinating the operation of these generators and loads can bring benefits in that the overall cost is reduced by own consumption or flexibility is provided to the distribution grid. However, these operation strategies are not yet state of the art, especially not in small and mediumsized enterprises. The model-based hardware-in-the-loop test concept that is presented in this work, supports the development of appropriate control technology for the automatisation of energy systems and processes. In this manner, control algorithms can be simulated in real-time, while being implemented on field devices including field communication. Functionalities and performance of the automation solution can be evaluated and flaws can be detected.
\end{abstract}

Keywords: hardware-in-the-loop, process control, virtual comissioning, renewable energy system

\section{Introduction}

It has become evident that future energy systems will have to cope with fluctuations in the energy supplied by regenerative sources. This could lead to temporary local congestions or overcapacities in the power distribution grid. Transmission and distribution network operators in Germany perform feed-in management to avoid congestions, curtailing the power input to the grid. Curtailment is basically undesirable as the plant owner has to be compensated for the deficit energy and renewable ressources remain unused. In 2017 the amount of curtailed energy in Germany summed up to $5,518 \mathrm{GWh}$ [1]. This number will increase with a higher penetration of wind and solar energy systems in the future.

Another way to stabilize the system is to design decentralized subsystems capable of adapting their feed-in and power consumption from the grid to the grid conditions. A key benefit of such energy subsystems is the flexibility that they offer to the distribution system operator (DSO) without the need of curtailment [2].

Modern production facilities often contain distributed renewable energy systems as well as loads that can be subject to demand side management, especially in small and medium- sized enterprises (SMEs) and municipal facilities. In order to achieve the desired flexibility, these energy subsystems have to take into account the nature of the power output from renewable energy sources: quasi-deterministic (predictable to a certain amount) from photovoltaics $(\mathrm{PV})$, controllable with constraints from combined heat and power (CHP) plants, and stochastic to quasi-deterministic from wind power. They also have to take into account constraints arising from different load types: continuous processes, discrete processes (e. g., purely on-off), essential loads, which cannot be switched off, less highly prioritized loads, which can be shut down at least for certain time periods, etc. The core task consists in matching the energy supply and demand by performing a demand-side scheduling and power dispatch within the decentralized energy subsystem. This may require local electrical energy storage units, such as batteries, because they serve both as load and as source.

The implementation of an energy subsystem as described above requires a local "intelligence", i. e., a controller. This controller receives measurement, status or prediction data from heterogeneous information sources via different interfaces and in different formats, processes them and outputs commands to the controlled units in the system. Many sophisti- 
cated proposals to solve this allocation problem using different methods can be found in the literature, e. g., multi-objective optimization [3] or rolling-horizon control [4]. Typically, these solutions are only validated by simulation. However, novel control algorithms cannot be implemented in the field without prior testing and characterization because untested control algorithms would pose a safety and security risk for equipment, processes, and environment. This is why we developed a hardware-in-the-loop (HiL) test concept for energy system control validation on field hardware including field communication.

\section{Methods}

To test and characterize the described automation solution for energy-optimized operation we created an HiL testbed. It consists of the controller hardware, field-communication peripherals, and a model of the controlled system. The functional diagram of the testbed is depicted in Fig. 1. The controller hardware is implemented as a programmable logic controller (PLC; Wago PFC200) because this is the state of the art for industrial process control. It is extended by an SQL database for data recording and a human machine interface $(\mathrm{HMI})$ in form of process visualization.

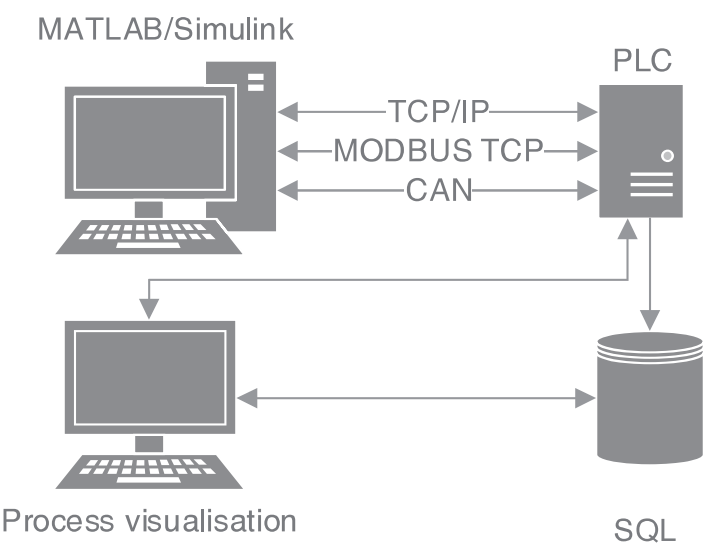

Fig. 1: Functional diagram of the HiL testbed

The controlled system with its many subsystems is modelled in Matlab/Simulink (Simscape Power Systems) on a desktop computer. The model environment communicates with the PLC via Modbus/TCP, CAN, and TCP/IP. The communication protocols are dictated by typical real-world environments in which the PLC is to operate.

The testbed is suited for controllers operating heterogeneous energy systems involving $A C$ and DC energy sources, loads, and processes in an energy-optimized manner.

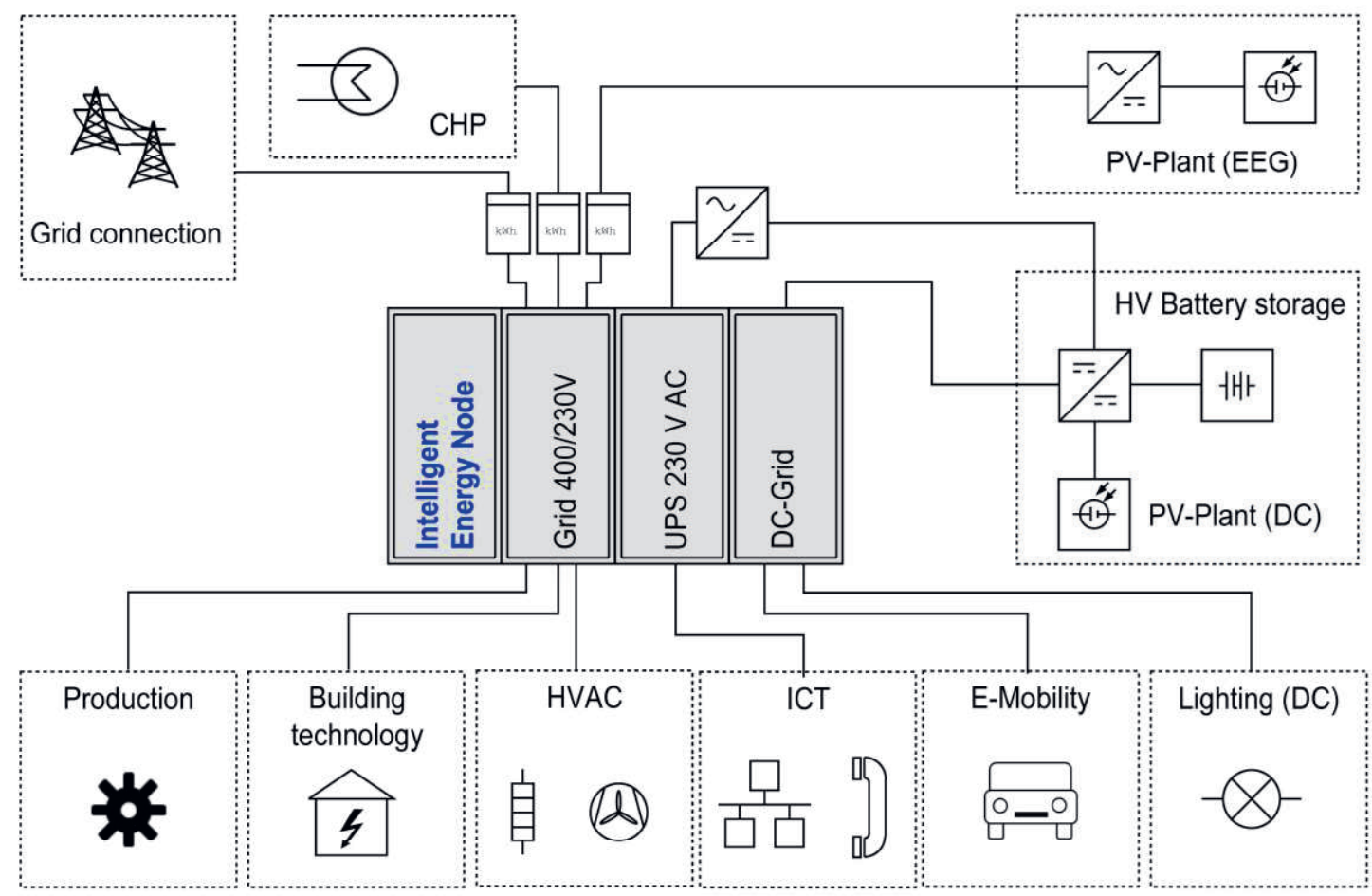

Fig. 2: Block diagram of an energy system containing typical components as well as a DC minigrid, which consists of DC loads, a high-voltage battery storage, and a DC-coupled photovoltaic power plant. 
A typical heterogenous energy system is depicted in Fig. 2 on the preceding page. The energy system is connected to the public electricity grid and has three operational voltage layers. The first layer is a conventional 400/230- $V$ three-phase AC grid, in which various $A C$ loads and sources are connected. As a second layer, the system contains a 350V DC subgrid, which is connected unidirectionally to the AC grid (consumption only). The DC subgrid is composed of a high-voltage battery storage, a PV power plant, and (partially refitted) DC loads. These loads are devices that run on DC internally, such as LED lighting and computers; operating them in a DC grid clearly reduces $A C / D C$ conversion losses.

The third layer is a single-phase AC grid unidirectionally connected to the DC subgrid (consumption only). It represents an uninterruptible power supply (UPS) for key components.

Figure 3 shows the top level of the modelled energy system. It is built in a modular way so that it can be adapted and parametrized for any given $\mathrm{AC}$ system comprising components belonging to one of six fundamental categories (1-6). The layers two (DC grid) and three (AC UPS) are implemented in submodels (grey and orange blocks). The same remark applies to the Modbus and CAN communication interfaces (yellow and blue blocks). The characte- ristics of the functional units used in the model have been derived from the characteristics of physical units that the PLC is to control in the field. To this end, historic data of representative energy system components were analyzed (PV system output, plant power consumption, peak powers, power distribution, etc.).

The control algorithm running on the PLC is rule-based and implemented according to IEC 61131. The many gaps in one's knowledge about the static and dynamic characteristics of system components and the limited resources available in field devices usually force one to get by without classical approaches such as PID control, state-space control, and controller parametrization by mathematical optimization.

Historic data and model-based PV power predictions are used to improve the scheduling of flexible loads and battery power. The implementation of $\mathrm{PV}$ power predictions is based on the python library "PVLIB-Python" [5] and the numerical weather prediction model "COSMOD2" of the German weather service "Deutscher Wetterdienst" (DWD) [6]. The prediction is updated every three hours with a forecast horizon of 27 hours and once a day with a 45-hour horizon. The forecast horizon of the power prediction is determined by the forecast horizon of the numerical weather prediction model.

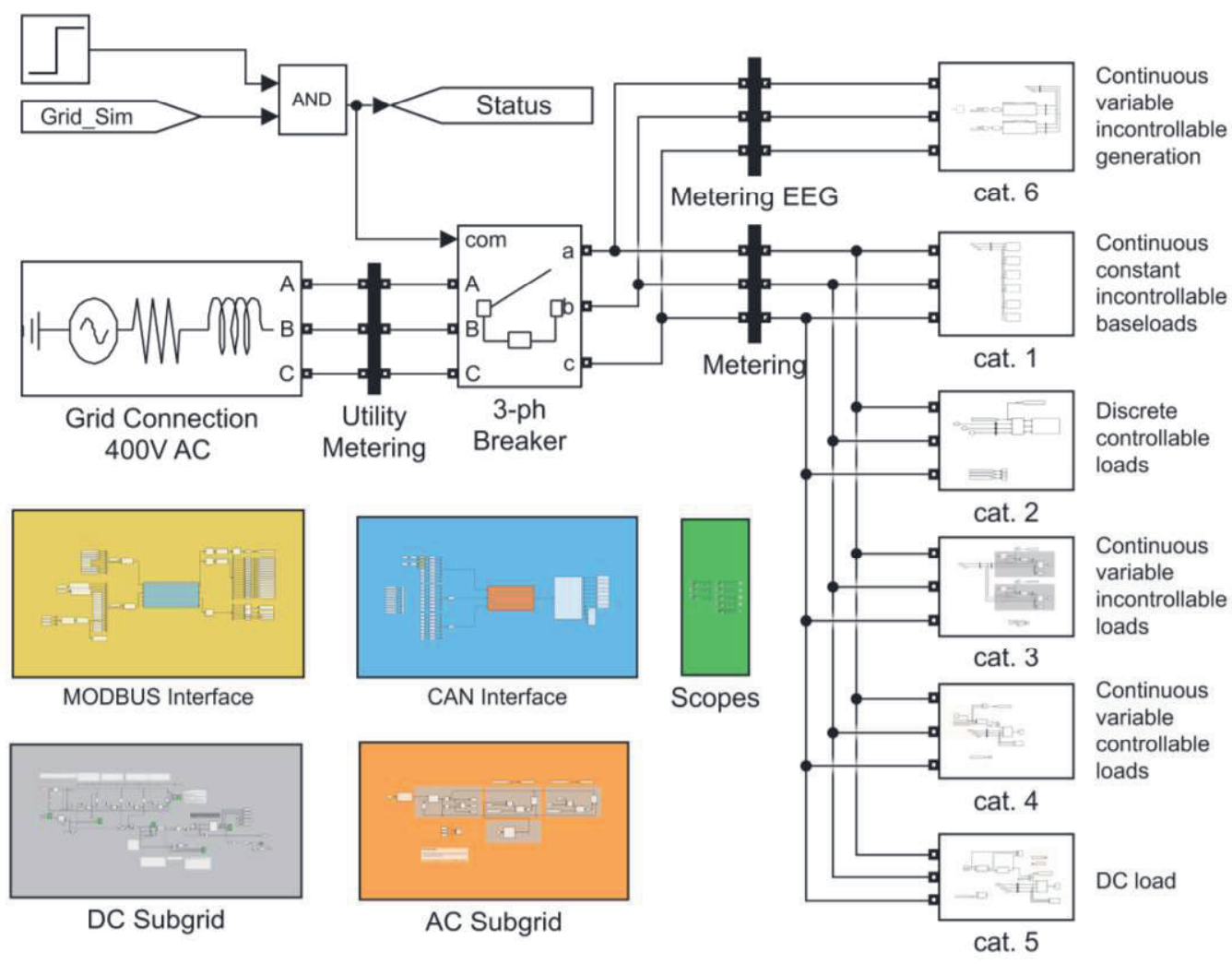

Fig. 3: Highest level of the energy system model for the real-time simulation and HiL test of the PLC-based system control. 


\section{Results}

The HiL test concept allows one to validate the control quality. Control algorithms can be tested and verified, while being implemented on field hardware and using field communication. The testing possibilities include control code errors, testing and validating of the system dynamics, consideration of special cases, and the simulation of critical states.

By unit testing, single programming organization units of the PLC code can be tested. By integration testing, modules of the energy system control, such as communication modules or device-specific modules, can be evaluated. By system testing, the entirety of the modeled (virtual-reality) components controlled by the PLC can be shown to better meet the objectives of load flexibility and cost reduction than the uncontrolled system.

Figure 4 shows the result of the HiL simulation of a daytime period of the energy system. A simple control algorithm was used for the energy system in order to facilitate the evaluation of the HiL testbed. The case shown demonstrates some typical features. Whereas PV excess power is available in the first half of the day and therefore has to be curtailed, this is not the case in the afternoon (Fig. 4a). The reason is that a $6-\mathrm{kW}$ load in the $\mathrm{AC}$ minigrid (layer 3) was switched on by the energysystem controller (Fig. 4b). As a consequence, the battery was charged in the morning (positive battery power) and discharged in the afternoon (negative battery power). This also shows in the state of charge of the battery (Fig. 4d). The resulting rectifier power at the input of the DC grid is presented in Fig. $4 \mathrm{e}$.

The chart in Fig. 5 on the following page is a comparison of predicted $\mathrm{PV}$ power and measured output power for an existing PV power plant. The prediction was based on a 45-hour wheather forecast horizon as described above and the measured data were taken on site by a field-measurement unit. As expected, the prediction does not agree perfectly with the actually observed PV power output; after all, wheather forecasts are uncertain. Nevertheless, even a partially correct prediction of $\mathrm{PV}$ output power to be expected on the next day is a valuable piece of information. It enables one to better run the energy system on the average (not in every single case) by increasing the own consumption of electrical energy from the PV power plant.

The charts in Fig. 6 show an analysis of exemplary historic energy-system data of two full days. During the morning hours of both (a)

(b)

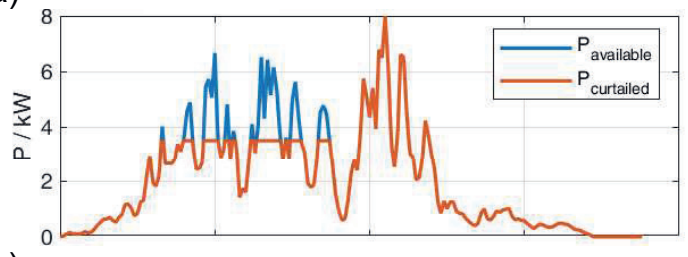

(c)
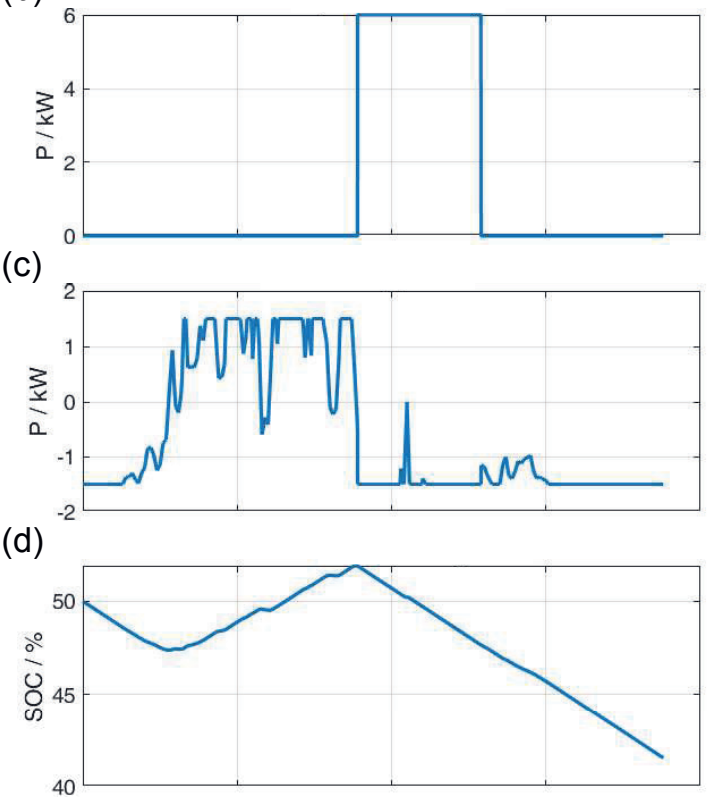

e)

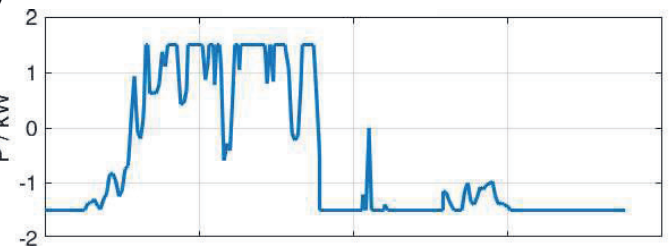

(d)

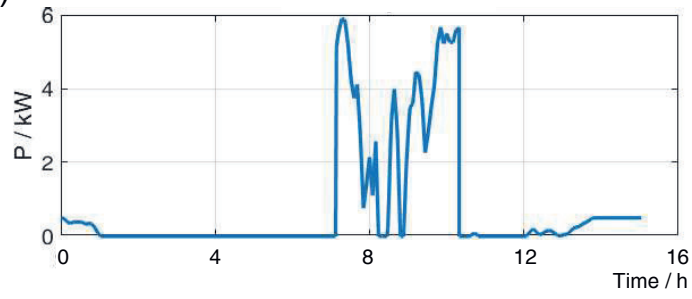

Fig. 4: Exemplary results of an energy system simulation. (a) Available (_) and curtailed (-) output power of a PV power plant. (b) Adaptive load adjustment in the AC minigrid (layer 3). (c) Resulting battery power in the DC minigrid (layer 2). (d) State of charge of the battery storage. (e) Rectifier power of the DC-grid feed-in from layer 1.

days the power demand in the energy system is higher than the PV power. During midday, the PV power exceeds the power demand. For this reason, the own consumption is limited to the instantaneous value of the power demand (Fig. 6a). As a result, power is drawn from the grid during all hours, except midday, where excess power is fed to the grid (Fig. 6b). This can also be derived from the 15-minute mean values, a common measure in energy economics for accounting purposes, of the load (Fig. $6 \mathrm{c}$ ) and residual grid powers (Fig. 6d). By controlling the single entities in the system in an intelligent way, the residual load curve can be 


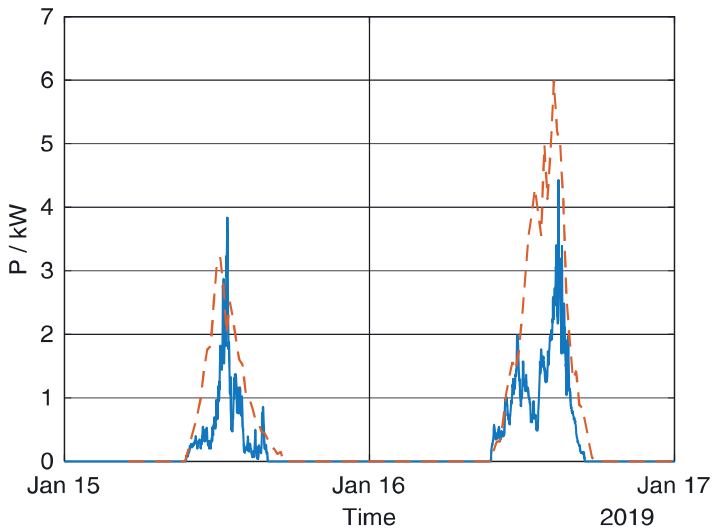

Fig. 5: $\quad$ Measured (-) and predicted (--) output power of an existing PV power plant (January $15^{\text {th }}$ and $16^{\text {th }}, 2019$ ). Prediction with a 45-hour forecast horizon based on numerical weather prediction.

(a)

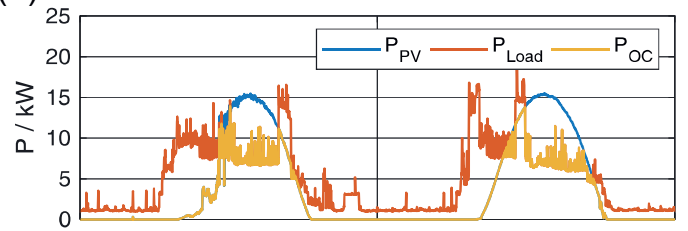

(b)

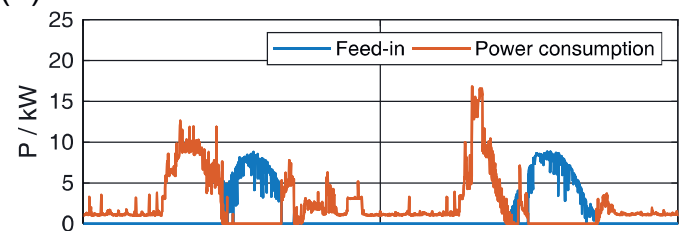

(c)

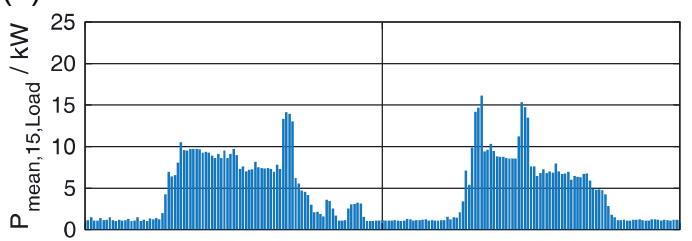

(d)

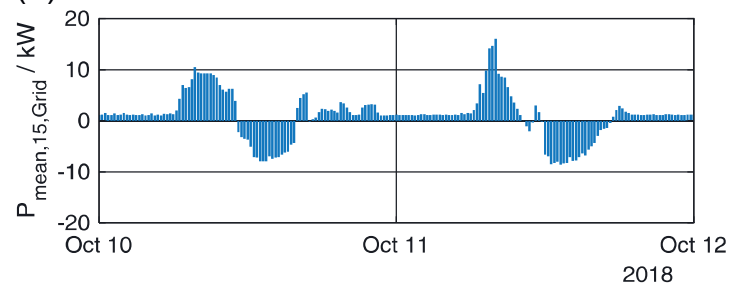

Fig. 6: Analysis of exemplary historic plant data (October 10 $0^{\text {th }}$ and 11th, 2018). (a) PV power (-), load (-) and own consumption (-). (b) Excess power fed to the grid (-), power consumption from the grid (-). (c) 15-minute mean values of power (load). (d) 15-minute mean values of power (grid). shaped in order to avoid load peaks, to offer flexibility to the grid, and to increase own consumption. This can be seen clearly, for instance, at the three peak load situations that arise from the charging of an electric vehicle, alongside the usual daily load curve.

\section{Discussion and Outlook}

The proposed testbed is suitable to characterize and test energy-optimized process control solutions that are implemented on typical industrial control hardware, including typical field communication in real-time. Different control algorithms can now be implemented on field hardware and their performance and implementation accuracy can be tested extensively. Furthermore a virtual commissioning based on VDI/VDE 3693 can be developed $[7,8]$.

The current control algorithm will be evaluated extensively with the developed simulation tool. Furthermore, the control algorithm will be extended and revised. Additionally the PV power prediction accuracy will be evaluated by comparison with measurement data of a long time period including all seasons.

\section{Acknowledgements}

This work was funded by the Central Innovation Programme for SMEs (ZIM) of the Federal Ministry for Economic Affairs and Energy (BMWi) - project number ZF4152303LF7.

\section{References}

[1] Bundesnetzagentur für Elektrizität, Gas, Telekommunikation, Post und Eisenbahnen, Quartalsbericht zu Netz- und Systemsicherheitsmaßnahmen, Gesamtjahr und Viertes Quartal 2017, Bonn (2018)

[2] J. Villar, R. Bessa, M. Matos, J. Electr. Pow. Syst. Res. 154, 329-340 (2018); doi: 10.1016/ j.epsr.2017.09.005

[3] A. Chaouachi et al., IEEE Trans. Indust. Electron. 60(4), 1688-1699 (2013); doi: 10. 1109/TIE.2012.2188873

[4] J. Silvente et al., Appl. Energy 155, 485-501 (2015)

[5] W. F. Holmgren, C. W. Hansen, M. A. Mikofski, J. Open Source Software 3(29), 884, (2018); doi: $10.21105 /$ joss. 00884

[6] M. Baldauf et al., Beschreibung des operationellen Kürzestfristvorhersagemodells COSMOD2 und COSMO-D2-EPS und seiner Ausgabe in die Datenbanken des DWD, Offenbach (2018)

[7] VDI/VDE 3693 Part 1, Virtual commissioning Model types and glossar (2016)

[8] VDI/VDE 3693 Part 2, Virtual commissioning Introduction of virtual commissioning in companies (2018) 